\title{
Human papillomavirus infection in women with and without cervical cancer in Nepal
}

\author{
Ang Tshering Lama Sherpa • Gary M. Clifford • Salvatore Vaccarella • \\ Sadhina Shrestha $\cdot$ Mari Nygård · Balman Singh Karki • Peter J. F. Snijders • \\ Chris J. L. M. Meijer · Silvia Franceschi
}

Received: 15 June 2009/Accepted: 29 October 2009/Published online: 22 November 2009

(C) Springer Science+Business Media B.V. 2009

\begin{abstract}
Objectives Cervical cancer is the most common malignancy among Nepalese women. Rational prevention measures are informed by epidemiological data on human papillomavirus (HPV) prevalence.

Methods Cervical specimens were obtained from 932 married women aged 15-59 years from the general population of Bharatpur, Nepal, as well as from 61 locally diagnosed invasive cervical cancers (ICC). HPV was detected using a GP5+/6+ PCR-based assay.

Results Among the general population, the overall prevalence of HPV was $8.6 \%$ (6.1\% for high-risk types). Prevalence of abnormal Pap smears was 3.6\%, including five high-grade squamous intraepithelial lesions. Residence in slum housing, lower education level, $\geq 3$ sexual partners in a woman's lifetime, and husband's extramarital affairs were significantly associated with HPV positivity. HPV
\end{abstract}

A. T. L. Sherpa · B. S. Karki

Kist Medical College, Lalitpur, Nepal

A. T. L. Sherpa

Section for International Health, University of Oslo, Oslo, Norway

G. M. Clifford $(\bowtie) \cdot S$. Vaccarella $\cdot$ S. Franceschi International Agency for Research on Cancer, 150 cours Albert Thomas, 69372 Lyon cedex 08, France e-mail: clifford@iarc.fr

S. Shrestha

B.P. Koirala Memorial Cancer Hospital, Bharatpur, Nepal

M. Nygård

Cancer Registry of Norway, Oslo, Norway

P. J. F. Snijders · C. J. L. M. Meijer

Vrije University Medical Center, Amsterdam, The Netherlands prevalence was relatively constant across all age groups. HPV16 was the most common type, both among the general population (1.9\%) and among 54 women with HPVpositive ICC (68.5\%). HPV18 (22.2\%) and 45 (5.6\%) were also common in ICC.

Conclusions Nepal has an intermediate burden of HPV infection, lower than many areas in India and China. Approximately $80 \%$ of cervical cancer in Nepal is theoretically preventable by HPV16/18 vaccines. In the meantime, screen-and-treat approaches should be encouraged to overcome difficulties that were encountered to recall women with screening-positive findings.

Keywords Cervical cancer - Human papillomavirus . Prevalence $\cdot$ Nepal

\begin{tabular}{ll}
\multicolumn{2}{l}{ Abbreviations } \\
CI & Confidence interval \\
HSIL & High-grade squamous intraepithelial lesions \\
HPV & Human papillomavirus \\
HPV16/18 & HPV16 and/or 18 \\
IARC & International Agency for Research on Cancer \\
ICC & Invasive cervical cancer \\
OR & Odds ratio
\end{tabular}

\section{Introduction}

Nepal is a land-locked country between India and China. Its 27 million inhabitants are divided approximately equally between the mountain/hills and the low plains bordering India. The adult literacy rate is estimated at $49 \%$ [1], and $24 \%$ of the population lives below the poverty line [2]. Life expectancy at birth is 61 years for men and women alike [3]. 
Cervical cancer is the most commonly reported malignancy among women in Nepal [4], although no populationbased estimates of cervical cancer incidence exist. Neither are there any data on the prevalence of high-risk human papillomavirus (HPV) infection in Nepal, which, in the absence of good-quality screening, is known to correlate with cervical cancer incidence [5].

The establishment of the viral etiology of cervical cancer has raised the hopes for primary and secondary prevention through HPV vaccination [6] and HPV DNA test-based screening $[7,8]$, respectively. The rationale and planning of such measures greatly benefit from knowledge of overall, age-, and type-specific HPV prevalence in women with and without cancer. To this end, the International Agency for Research on Cancer (IARC) has carried out surveys in representative samples of women worldwide [9].

The purpose of the study was to assess the prevalence of type-specific HPV infection in the general female population and in invasive cervical cancer (ICC) in Nepal.

\section{Materials and methods}

\section{HPV prevalence survey}

Study methods were similar to those used for previous IARC HPV prevalence surveys [9]. The study area included four semi-urban districts (Ganesthan, Muktinagar, Naurange and Bhojad) of Bharatpur municipality, Chitawan Province, in the low plains of Nepal. The study purpose was to enroll approximately 100 women from the general population in each five-year age group between 15-19 and 54-59 years. All mentally and physically competent women aged 15-59 years were eligible for the study, regardless of their marital status. All women were enumerated at their homes by local community workers and invited to the study clinic, which was set up in the community specifically for the present study between October 2006 and March 2007.

Of the 1,606 invited women, 514 (32.0\%) did not accept the invitation, including 42 who had never been married. Refusal rates were $47,13,10$, and $49 \%$ among women aged 15-24, 25-34, 35-44, and 45-59 years, respectively. The most common reasons for non-participation were lack of perception of the need to undergo screening in the absence of symptoms $(n=195)$ and lack of permission of husband $(n=63)$. In addition, among the 1,092 women who accepted the invitation to come to the study clinic, 113 eventually refused to undergo a pelvic examination, (including 54 unmarried women who reported themselves to be virgins and 18 pregnant women) and hence were unable to provide a cervical cell specimen. In the end, no unmarried woman provided a cervical cell specimen.
An interview was administered by one of two female interviewers in the local dialect. The structured questionnaire included information on socio-demographic characteristics, reproductive and menstrual factors, sexual habits of women and their husbands, and lifetime use of contraceptive methods.

A total of 979 women underwent a pelvic examination by a midwife or female doctor. After the preparation of a conventional Pap smear, a sample of exfoliated cervical cells from the endocervix and ectocervix was collected with a cervexbrush (Rovers Medical Devices B.V., Oss, the Netherlands). After being inserted into the endocervical canal and rotated gently $180^{\circ}$, the brush containing cellular material was placed in a vial containing PreservCyt media (Hologic, Inc., Marlborough, MA, USA).

Pap smears were read by a pathologist (S.S.) at the B.P. Koirala Memorial Cancer Hospital, Bharatpur, the national referral centre for all cancer in Nepal.

\section{Women with ICC}

Tumor biopsies, formalin fixed and embedded in paraffin, were retrieved for all women $(n=68)$ with histologically confirmed ICC to B.P. Koirala Memorial Cancer Hospital during 2006.

\section{Ethical approval}

All participants for the HPV prevalence survey signed informed consent forms according to the recommendations of the IARC and the B.P. Koirala Memorial Cancer Hospital ethical review committees, both of which approved the present study.

\section{HPV detection}

HPV testing was performed on exfoliated cervical cells and ICC biopsies at the Department of Pathology at the Vrije University Medical Center, Amsterdam, The Netherlands. DNA was extracted from the PreservCyt sample using magnetic beads (Macherey-Nagel, Düren, Germany) on a robotic system (Hamilton, Germany), according to the manufacturer's instructions. ICC biopsies were sectioned using a 'sandwich' approach, whereby inner tumor sections were destined for HPV testing and outer sections for histological confirmation of tumor tissue. One or more five-lM sections representing approximately $1 \mathrm{~cm}^{2}$ of tissue were predigested with proteinase $\mathrm{K}$ after which DNA was extracted using magnetic beads (Macherey-Nagel).

Beta-globin PCR analysis was performed firstly to confirm the presence of human DNA in all specimens [10]. The overall presence of HPV DNA was determined by performing a general primer GP5+/6+ -mediated PCR, 
which permits the detection of a broad spectrum of genital HPV types at the subpicogram level [11]. HPV positivity was assessed by hybridization of PCR products in an enzyme immunoassay using two HPV oligoprobe cocktails which, together, detect the following 44 HPV types: HPV6, $11,16,18,26,30,31,32,33,34,35,39,40,42,43,44,45$, 51, 52, 53, 54, 55, 56, 57, 58, 59, 61, 64, 66, 67, 68, 69, 70, 71 (equivalent to $\mathrm{CP} 8061$ ), 72, 73, 81 (equivalent to CP8304), 82 (IS39 and MM4 subtypes), 83 (equivalent to MM7), 84 (equivalent to MM8), cand85, 86, cand89 (equivalent to CP6108), and JC9710. Subsequent HPV typing was performed by reverse-line blot hybridization of PCR products, as described previously [12]. HPV types considered high-risk for this analysis included HPV16, 18, $31,33,35,39,45,51,52,56,58,59,68,73$, and 82 [13]. All other HPV types were considered low-risk.

\section{Statistical analysis}

Crude, age-stratified, and age-standardized (world standard population [14]) HPV prevalence with $95 \%$ confidence intervals (CIs) was estimated.

Odds ratios (ORs) for HPV positivity and corresponding $95 \%$ CIs were calculated by means of unconditional logistic regression equations adjusted for age $(<25,25-34$, 35-44, and 45-59 years). Adjustment for additional variables was also performed, but not shown. The statistical significance of trends for ORs was assessed by considering the categorical variables as a continuous variable in the logistic model.

Prevalence ratios and corresponding 95\% CIs were used to compare the relative frequency of the most common HPV types in HPV-positive ICC with that among cytologically normal HPV-positive women from the HPV prevalence survey.

\section{Results}

\section{HPV prevalence survey}

Of 979 married women who provided cervical cell samples, 36 had inadequate HPV DNA results (i.e., beta-globin PCR negative), and an additional 11 had inadequate cytology, leaving 932 women with valid results for both HPV and cytology. Among them, 34 (3.6\%) had abnormal cytological findings, including 29 (3.1\%) atypical squamous cells of undetermined significance/low-grade squamous intraepithelial lesions, and five $(0.5 \%)$ high-grade squamous intraepithelial lesions (HSIL).

Overall HPV prevalence was $8.6 \%$, being 20.6 and $8.1 \%$ among women with and without cervical abnormalities, respectively (Table 1). The corresponding prevalence age- standardized to the world population was also $8.6 \%$. In total, $64(6.9 \%)$ women had single-type infections, and 16 $(1.7 \%)$ had multiple-type infections. Prevalence of highrisk types was $6.1 \%$, compared to $3.8 \%$ for low-risk types. The most common high-risk types among women with normal cytology were HPV16 (1.5\%), 58 (0.9\%), 56 $(0.7 \%), 18(0.6 \%)$, and $52(0.5 \%)$. HPV42 $(0.8 \%)$ was the most commonly detected low-risk type. HPV16 also predominated among women with cervical abnormalities, including four out of five HSIL.

Figure 1 shows the age-specific prevalence of HPV, classified hierarchically into 1) HPV16 and/or 18 (HPV16/ 18), 2) other high-risk types and [3] low-risk types only. Overall, HPV prevalence was not significantly different by age group. However, the prevalence of HPV16/18 increased from $1.1 \%$ among women aged $15-24$ years to $5.2 \%$ among women aged 45-59 years ( $p$ for trend by age group $=0.02$ ). Finer stratification of women aged less than 25 years revealed overall HPV prevalences of 8.9, 6.9, and $7.2 \%$ among women aged less than 20, 20-22, and 23-24 years, respectively.

Table 2 shows the relationship between HPV positivity and various characteristics of study participants, adjusted for age. Significant differences in HPV positivity were observed for residence in slum housing $(\mathrm{OR}=2.96 ; 95 \%$ CI: 1.76-4.98), education level (OR for none versus higher $=2.41 ; 95 \% \mathrm{CI}: 1.04-5.56)$, lifetime number of sexual partners (OR for $\geq 3$ vs. 1 partner $=4.09$; $95 \% \mathrm{CI}$ : 1.52-11.01), reporting of husband's extramarital sexual relationships $(\mathrm{OR}=1.71 ; 95 \% \mathrm{CI}: 1.04-2.80)$, and lack of history of cervical cancer screening $(\mathrm{OR}=1.88 ; 95 \% \mathrm{CI}$ : 1.01-3.51). These associations remained materially unchanged when additionally adjusted for residence in slum housing, education level, and/or lifetime number of sexual partners, as appropriate (data not shown).

Religion, occupation, age at first marriage, age at first sexual intercourse, and number of births were not significantly related to HPV positivity in either adjustment model (Table 2) and neither were smoking or chewing tobacco habits (reported by 18.6 and $6.2 \%$ of women, respectively), hormonal contraceptive use (reported by $43.4 \%$ of women), condom use $(18.3 \%)$, intrauterine device use $(5.1 \%)$, or a history of spontaneous $(26.5 \%)$ or induced abortions (26.0\%) (data not shown).

\section{Women with ICC}

Biopsies that were beta-globin PCR negative $(n=5)$ or without histological evidence of tumor $(n=2)$ were excluded. Among the remaining 61 ICC biopsies, six were adenocarcinoma, and the rest were squamous-cell carcinoma. The median age of women with ICC was 50 years (range: 29-60 years). Seven squamous-cell carcinoma 
Table 1 Prevalence of human papillomavirus (HPV) types by cytological findings and overall among 932 married women. Bharatpur, Nepal, 2006-2007

\begin{tabular}{|c|c|c|c|c|c|c|c|c|c|}
\hline \multirow[t]{2}{*}{ HPV type } & \multicolumn{3}{|c|}{ Normal cytology $(n=898)$} & \multicolumn{3}{|c|}{ Abnormal cytology $(n=34)$} & \multicolumn{3}{|c|}{ Total $(n=932)$} \\
\hline & Single & Multiple & Total $(\%)$ & Single & Multiple & Total $(\%)$ & Single & Multiple & Total $(\%)$ \\
\hline $\mathrm{HPV}-$ & - & - & $825(91.9)$ & - & - & $27(79.4)^{\mathrm{a}}$ & - & - & $852(91.4)$ \\
\hline $\mathrm{HPV}+$ & 59 & 14 & $73(8.1)$ & 5 & 2 & $7(20.6)$ & 64 & 16 & $80(8.6)$ \\
\hline HR HPV+ & 37 & 13 & $50(5.6)$ & 5 & 2 & $7(20.6)$ & 42 & 15 & $57(6.1)$ \\
\hline LR HPV+ & 22 & 11 & $33(3.7)$ & 0 & 2 & $2(5.9)$ & 22 & 13 & $35(3.8)$ \\
\hline \multicolumn{10}{|c|}{ High-risk infections } \\
\hline 16 & 10 & 3 & $13(1.5)$ & $3^{\mathrm{b}}$ & $2^{\mathrm{b}}$ & $5(14.7)$ & 13 & 5 & $18(1.9)$ \\
\hline 18 & 4 & 1 & $5(0.6)$ & 0 & 0 & 0 & 4 & 1 & $5(0.5)$ \\
\hline 31 & 1 & 0 & $1(0.1)$ & 1 & 0 & $1(2.9)$ & 2 & 0 & $2(0.2)$ \\
\hline 33 & 2 & 0 & $2(0.2)$ & 0 & 0 & 0 & 2 & 0 & $2(0.2)$ \\
\hline 35 & 2 & 1 & $3(0.3)$ & 0 & 0 & 0 & 2 & 1 & $3(0.3)$ \\
\hline 39 & 4 & 0 & $4(0.5)$ & 0 & 0 & 0 & 4 & 0 & $4(0.4)$ \\
\hline 45 & 0 & 2 & $2(0.2)$ & 0 & 0 & 0 & 0 & 2 & $2(0.2)$ \\
\hline 51 & 2 & 0 & $2(0.2)$ & 0 & 0 & 0 & 2 & 0 & $2(0.2)$ \\
\hline 52 & 2 & 2 & $4(0.5)$ & 1 & 0 & $1(2.9)$ & 3 & 2 & $5(0.5)$ \\
\hline 56 & 2 & 4 & $6(0.7)$ & 0 & 0 & 0 & 2 & 4 & $6(0.6)$ \\
\hline 58 & 6 & 2 & $8(0.9)$ & 0 & 0 & 0 & 6 & 2 & $8(0.9)$ \\
\hline 59 & 0 & 0 & 0 & 0 & 0 & 0 & 0 & 0 & 0 \\
\hline 68 & 0 & 1 & $1(0.1)$ & 0 & 0 & 0 & 0 & 1 & $1(0.1)$ \\
\hline 73 & 2 & 0 & $2(0.2)$ & 0 & 0 & 0 & 2 & 0 & $2(0.2)$ \\
\hline 82 & 0 & 1 & $1(0.1)$ & 0 & 0 & 0 & 0 & 1 & $1(0.1)$ \\
\hline Subtotal & 37 & 17 & $54-$ & 5 & 2 & $7-$ & 42 & 19 & $61-$ \\
\hline \multicolumn{10}{|c|}{ Low-risk infections } \\
\hline 6 & 2 & 0 & $2(0.2)$ & 0 & 0 & 0 & 2 & 0 & $2(0.2)$ \\
\hline 11 & 0 & 0 & 0 & 0 & 0 & 0 & 0 & 0 & 0 \\
\hline 26 & 0 & 0 & 0 & 0 & 0 & 0 & 0 & 0 & 0 \\
\hline 30 & 2 & 0 & $2(0.2)$ & 0 & 0 & 0 & 2 & 0 & $2(0.2)$ \\
\hline 34 & 0 & 0 & 0 & 0 & 0 & 0 & 0 & 0 & 0 \\
\hline 40 & 0 & 0 & 0 & 0 & 0 & 0 & 0 & 0 & 0 \\
\hline 42 & 2 & 5 & $7(0.8)$ & 0 & $1^{\mathrm{a}}$ & $1(2.9)$ & 2 & 6 & $8(0.9)$ \\
\hline 43 & 0 & 1 & $1(0.1)$ & 0 & 0 & 0 & 0 & 1 & $1(0.1)$ \\
\hline 44 & 0 & 0 & 0 & 0 & 0 & 0 & 0 & 0 & 0 \\
\hline 53 & 0 & 0 & 0 & 0 & 0 & 0 & 0 & 0 & 0 \\
\hline 54 & 0 & 0 & 0 & 0 & 0 & 0 & 0 & 0 & 0 \\
\hline 55 & 1 & 0 & $1(0.1)$ & 0 & 0 & 0 & 1 & 0 & $1(0.1)$ \\
\hline 61 & 0 & 0 & 0 & 0 & 0 & 0 & 0 & 0 & 0 \\
\hline 64 & 0 & 0 & 0 & 0 & 0 & 0 & 0 & 0 & 0 \\
\hline 66 & 0 & 0 & 0 & 0 & 0 & 0 & 0 & 0 & 0 \\
\hline 67 & 1 & 3 & $4(0.5)$ & 0 & 0 & 0 & 1 & 3 & $4(0.4)$ \\
\hline 69 & 2 & 0 & $2(0.2)$ & 0 & 0 & 0 & 2 & 0 & $2(0.2)$ \\
\hline 70 & 4 & 0 & $4(0.5)$ & 0 & 0 & 0 & 4 & 0 & $4(0.4)$ \\
\hline 72 & 0 & 0 & 0 & 0 & 0 & 0 & 0 & 0 & 0 \\
\hline 81 & 1 & 0 & $1(0.1)$ & 0 & 0 & 0 & 1 & 0 & $1(0.1)$ \\
\hline 86 & 3 & 0 & $3(0.3)$ & 0 & 0 & 0 & 3 & 0 & $3(0.3)$ \\
\hline CP6108 & 1 & 0 & $1(0.1)$ & 0 & 0 & 0 & 1 & 0 & $1(0.1)$ \\
\hline JC9710 & 2 & 4 & $6(0.7)$ & 0 & $1^{\mathrm{a}}$ & $1(2.9)$ & 2 & 5 & $7(0.8)$ \\
\hline Subtotal & 21 & 13 & $34-$ & 0 & 2 & $2-$ & 21 & 15 & $36-$ \\
\hline$X$ & 1 & 0 & $1(0.1)$ & 0 & 0 & 0 & 1 & 0 & $1(0.1)$ \\
\hline Total infections & 59 & 30 & $89-$ & 5 & 4 & $9-$ & 64 & 34 & $98-$ \\
\hline
\end{tabular}

$X$ uncharacterized type

a One high-grade squamous intraepithelial lesion (HSIL)

b Two HSIL 


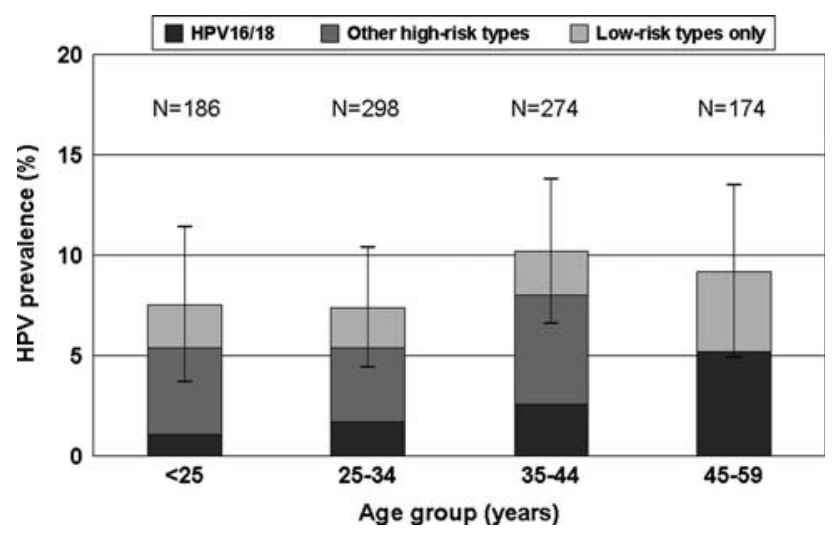

Fig. 1 Age-specific prevalence of human papillomavirus (HPV) DNA by HPV type(s) among 932 married women. Bharatpur, Nepal, 2006-2007

biopsies were HPV negative. The comparison of typespecific HPV prevalence in the 54 women with HPVpositive ICC and the $73 \mathrm{HPV}$-positive women with normal cytology from the HPV prevalence survey is shown in Table 3. HPV16 was found in $37(68.5 \%)$ HPV-positive ICC biopsies. The next most common types were HPV18 $(22.2 \%)$ and $45(5.6 \%)$. Prevalence ratios in women with ICC versus those with normal cytology were 3.85 (95\% CI: 2.79-5.32) for HPV16; 3.24 (95\% CI: 1.84-5.71) for HPV18; 2.03 (95\% CI: 0.65-6.20) for HPV45; and 0.13 (95\% CI: 0.04-0.39) for the combination of other high-risk types. Multiple-type infections were much less common in HPV-positive women with ICC than in HPV-positive women with normal cytology (Table 3).

\section{Discussion}

The major finding of this present study, the first undertaken in Nepal, is that the burden of HPV infection in the general population $(8.6 \%)$ is somewhat lower than that observed in previous studies performed using the same HPV testing protocol in neighboring countries (e.g., 17\% in Tamil Nadu, India, [15] and 15-18\% range in three provinces in China [16-18]), and much less than in the high-risk areas in Asia and Africa (e.g., 35\% in Mongolia [19] and 51\% in Guinea [20]). Nevertheless, HPV prevalence remained greater than that in other areas in Asia (e.g., 2\% in Hanoi, Vietnam [21], 4\% in Songkla, Thailand [22]), and in Europe [23].

One of the other main aims of the IARC HPV prevalence surveys is to describe the variations of age- and typespecific HPV prevalence by geographical region. The agespecific curve of HPV prevalence in Nepal resembled the flat age curves reported previously in some low-resource countries in Asia and Africa [15-18, 24], albeit at a lower level of HPV prevalence. It clearly differed, on the other hand, from the steep decrease in HPV prevalence seen by age in high- and medium-resource countries in Europe, the Americas, and Asia [23, 25].

As in previous IARC HPV prevalence surveys in Asia [15-18], the gynecological exam was acceptable only to married women, so, the HPV prevalence estimate represents that in married women only. Nevertheless, age at first marriage $($ median $=17$ years, intraquartile range 15-19) was relatively early, and $91 \%$ of participants reported an age at first sexual intercourse that was the same as, or greater than, age at first marriage, suggesting that marriage is a good proxy for sexual activity in this population. HPV16/18 prevalence, however, was highest among older women. HPV16/18 infections in older women are more likely to represent persistent infections [26] and are more likely to progress to cancer than other high-risk types [27].

As expected, the strongest risk factors for HPV positivity were confirmed, as elsewhere [28], to be the sexual behavior of women and their husbands. Nevertheless, both the percentage of women who reported two sexual partners or more in their lifetime $(9.9 \%)$ and the prevalence of husband's extramarital sexual relationships (32.1\%) were relatively low compared to similar surveys in other geographical areas (ranges: 6-64 and 12-100\%, respectively) [16-19, 28, 29]. A fourfold elevated risk of HPV positivity was, however, detected among the $2.6 \%$ of women who reported three sexual partners or more in their lifetime, which included at least four known commercial sex workers.

Residence in slum housing, low education level, and absence of previous cervical cancer screening were also associated with HPV positivity in this population, even after adjustment for sexual behavior, suggesting some association between lower socio-economic status and HPV prevalence in Nepal. Of note, the relationship between education level and HPV prevalence in previous IARC surveys in Asia included inverse [25] and direct associations [21, 22] as well as no relationship [16].

A pilot project of screening by visual inspection of 5,000 women was undertaken at the B.P. Koirala Memorial Cancer Hospital between 2003 and 2005. Therefore, the reported percentage of previous cervical cancer screening in our present study (24.6\%) is expected to be much higher than the national average, and to largely reflect a history of visual inspection.

As in most previous similar studies, HPV16 was confirmed to be the most common type in the whole range of women considered, i.e., $1.5,14.7$, and $68.5 \%$, respectively, in cytologically normal and abnormal women and in women with HPV-positive ICC [9, 30]. HPV52 and 58, however, were not as predominant as in similar surveys in China [16-18]. We also confirmed that the type distribution 
Table 2 Odds ratios (ORs) for human papillomavirus (HPV) positivity and corresponding 95\% confidence intervals (CIs) according to selected characteristics among 932 married women. Bharatpur, Nepal, 2006-2007
${ }^{a}$ Some figures do not add up to the total because of few missing ${ }^{b}$ Adjusted for age

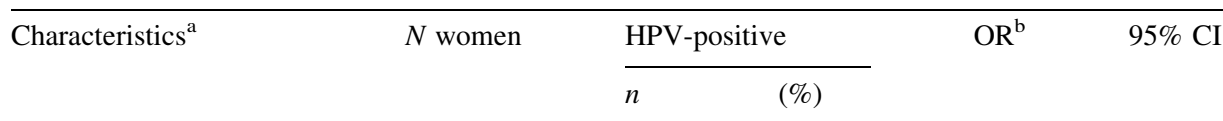

Age (years)

$<25$

25-34

186

298

(7.5)

(7.4)

1

0.98

$35-44$

(10.2)

1,40

(0.49-1.97)

45-59

174

(9.2)

$\chi_{1}^{2}$ for trend

1.24

$(0.72-2.73)$

Religion

Hindu

789

0.98

(0.59-2.63)

Buddhist

(8.0)

Other

1

1.69

$p=0.321$

Occupation

Unemployed

$(7.7)$

0.96

(0.92-3.08)

26

2

744

(9.0)

Employed

188

67

13

(6.9)

1

0.74

(0.40-1.38)

Residence in slum housing

No

798

(7.0)

Yes

134

(17.9)

1

2.96

$(1.76-4.98)$

Education level

Higher than secondary

Primary/secondary

147

None

(10.9)

$\chi^{2}{ }_{1}$ for trend

424

27

46

Age at 1st marriage

$\begin{array}{lll}\geq 19 & 280 & 19 \\ 16-18 & 352 & 33 \\ \leq 15 & 299 & 28\end{array}$

(9.4)

(9.4)

$$
1
$$

1.65

$\chi^{2}{ }_{1}$ for trend

28

Age at 1st intercourse

$\begin{array}{ll}\geq 19 & 286 \\ 16-18 & 373 \\ \leq 15 & 269\end{array}$

19

33

28

$\chi_{1}^{2}$ for trend

2.41

(0.70-3.90)

5.24

(1.04-5.56)

$p=0.022$

Lifetime number of sexual partners

$\begin{array}{lr}1 & 828 \\ 2 & 67 \\ \geq 3 & 24 \\ \chi^{2}{ }_{1} \text { for trend } & \end{array}$

68

5

6

Husband's extramarital sexual relationships

$\begin{array}{lll}\text { No } & 588 & 40 \\ \text { Yes } & 278 & 3\end{array}$

40

(6.6)

1

1.44

(0.79-2.62)

1.42

$(0.76-2.66)$

1.09

$p=0.297$

History of cervical cancer screening

$\begin{array}{ll}\text { Yes } & 229 \\ \text { No } & 703\end{array}$

13

67

Number of births

0

$1-2$

3-5

$\geq 6$

703

(6.8)

(11.2)

1

1.38

1.67

$(0.76-2.50)$

$(0.89-3.11)$

2.59

$p=0.108$

1

0.90

4.09

$(0.35-2.32)$

4.56

(1.52-11.01)

$\chi^{2}{ }_{1}$ for trend

$\begin{array}{rr}45 & 4 \\ 472 & 38 \\ 322 & 27 \\ 92 & 11\end{array}$

(8.9)

(8.1)

(8.4)

(12.0)

1

1.88

(1.01-3.51)

1.11

(0.37-3.29)

1

0.94

1.39

$(0.54-1.65)$

0.18

(0.63-3.05)

$p=0.674$ 
Table 3 Prevalence of selected human papillomavirus (HPV) types in 54 women with HPV-positive invasive cervical carcinomas (ICC) ${ }^{\mathrm{a}}$ and 73 HPV-positive women with normal cytology. Bharatpur, Nepal, 2006-2007

\begin{tabular}{llcc}
\hline HPV type & $\begin{array}{l}\text { ICC }(n=54) \\
\text { Total }(\%)\end{array}$ & $\begin{array}{l}\text { Normal cytology }(n=73) \\
\text { Total }(\%)\end{array}$ & $\begin{array}{l}\text { ICC:Normal cytology } \\
\text { Prevalence ratio }(95 \% \text { CI })\end{array}$ \\
\hline 16 & $37(68.5)$ & $13(17.8)$ & $3.85(2.79-5.32)$ \\
18 & $12(22.2)$ & $5(6.8)$ & $3.24(1.84-5.71)$ \\
45 & $3(5.6)$ & $2(2.7)$ & $2.03(0.65-6.20)$ \\
Other high-risk types & $3^{\mathrm{b}}(5.6)$ & $32(43.8)$ & $0.13(0.04-0.39)$ \\
Any low-risk type & 0 & $32(43.8)$ & $0(0-0.17)^{\mathrm{d}}$ \\
$X$ & 0 & $1(1.4)$ & $0(0-52.72)^{\mathrm{d}}$ \\
Multiple infections & $1^{\mathrm{c}}(1.9)$ & $14(19.2)$ & $0.10(0.01-0.68)$ \\
\hline
\end{tabular}

$X$ uncharacterized type

CI confidence interval

${ }^{a}$ Seven HPV-negative cancers are not included

b One HPV31, one HPV33, and one HPV56

${ }^{c}$ HPV $16+18$

${ }^{\mathrm{d}}$ Exact $95 \%$ CIs

among the general population was not representative of that in women with cervical cancer from the same region, with the relative importance of HPV16/18 being much greater in ICC, accounting for a total of $89 \%$ (95\% CI: 77$96 \%$ ) of HPV-positive cases. If seven (probably falsely) HPV-negative ICC were included in the denominator, HPV16/18-related ICC would represent 79\% (95\% CI: 66$88 \%$ ) of cases. This is consistent with the findings of a meta-analysis, which reported that the fraction of ICC attributable to HPV16/18 may be higher in the Indian subcontinent than in China and other parts of East Asia, and similar to that in Europe and the United States [30]. Furthermore, although multiple infections were frequent in the general population, $98.1 \%$ of HPV-positive ICC were infected with a single HPV type, highlighting the selection of one high-risk type in the course of malignant transformation.

Major strengths of our present study include a large and population-based sample, the use of a standardized and well-validated HPV test allowing comparisons with similar studies around the world [9] and the presence of a concurrent series of women with ICC drawn from the same study area. An overall participation rate of $70 \%$ was also relatively high compared to previous similar studies in Asia. However, there was a differential participation rate by age, being lower in the extreme age groups. Although HPV infection is asymptomatic, it is possible that lack of participation could have affected HPV prevalence estimates. The comparisons relative to other worldwide regions remain consistent, however, even when restricted to women aged 25-44 years, for whom participation approached $90 \%$ in Nepal.
No large-scale cytology-based screening programs have been performed in Nepal. Therefore, problems of sensitivity and specificity in Pap smear reading are likely to have existed, as suggested by the low HPV prevalence (20\%) among women with cytological abnormalities. Detection of high-risk HPV DNA in HSIL, however, was consistent with findings from elsewhere.

Finally, despite the availability of a fund to cover all diagnostic and treatment costs, and repeated insistence by the study staff, the greatest disappointment of the study was the refusal of the women with HSIL to be referred to colposcopy for diagnostic follow-up. This refusal might have been related to delays incurred by the political unrest in Nepal in 2006-2007 and some rumors of bleeding and pain from women who had undergone biopsy and/or cryotherapy in previous pilot screening programs. Although efforts to follow up screening-positive women continue, our experience highlights once again [31] how lack of familiarity with the usefulness of screening and the treatable nature of cervical cancer can act as strong barriers to the accomplishment of complete follow-up and treatment of screening-detected cervical lesions.

In conclusion, the prevalence of HPV infection in the general population would suggest that Nepal, where cancer registration is not available, is a country with intermediate cervical cancer risk. Screen-and-treat approaches should be encouraged to overcome the difficulties encountered in the recall of women $[32,33]$. The care HPV test, for example, appears particularly promising [7]. In respect to the potential benefit of vaccination, approximately $80 \%$ of cervical cancer in Nepal would be prevented by a vaccine including HPV16/18. 
Acknowledgments The authors of the manuscript have no conflict of interest to declare. The authors thank Dr Johanne Sundby for valuable comments.

Financial support This work was supported by the Bill \& Melinda Gates Foundation (grant number 35537).

\section{References}

1. United Nations Educational Scientific and Cultural Organization (2006) EFA Global Monitoring Report. Available from URL: http://gmr.uis.unesco.org/selectindicators.aspx

2. The World Bank Group (2006) 2006 World Development Indicators. Table 2.7: Poverty. Available from URL: http://devdata. worldbank.org/wdi2006/contents/Section2.htm

3. World Health Organization (2006) The world health report 2006: working together for health. World Health Organization, Geneva

4. B.P. Koirala Memorial Cancer Hospital (Nepal) (2004) Annual report 2003. B.P. Koirala Memorial Cancer Hospital, Chitawan

5. Maucort-Boulch D, Franceschi S, Plummer M (2008) International correlation between human papillomavirus prevalence and cervical cancer incidence. Cancer Epidemiol Biomarkers Prev 17:717-720

6. Kahn JA, Burk RD (2007) Papillomavirus vaccines in perspective. Lancet 369:2135-2137

7. Qiao YL, Sellors JW, Eder PS et al (2008) A new HPV-DNA test for cervical-cancer screening in developing regions: a crosssectional study of clinical accuracy in rural China. Lancet Oncol 9:929-936

8. Sankaranarayanan R, Nene BM, Shastri SS et al (2009) HPV screening for cervical cancer in rural India. N Engl J Med 360:1385-1394

9. Clifford GM, Gallus S, Herrero R et al (2005) Worldwide distribution of human papillomavirus types in cytologically normal women in the International Agency for Research on Cancer HPV prevalence surveys: a pooled analysis. Lancet 366:991-998

10. Roda Husman AM, Snijders PJ, Stel HV, van den Brule AJ, Meijer CJ, Walboomers JM (1995) Processing of long-stored archival cervical smears for human papillomavirus detection by the polymerase chain reaction. Br J Cancer 72:412-417

11. Jacobs MV, Walboomers JM, Snijders PJ et al (2000) Distribution of 37 mucosotropic HPV types in women with cytologically normal cervical smears: the age-related patterns for high-risk and low-risk types. Int J Cancer 87:221-227

12. van den Brule AJ, Pol R, Fransen-Daalmeijer N, Schouls LM, Meijer CJ, Snijders PJ (2002) GP5+/6+ PCR followed by reverse line blot analysis enables rapid and high-throughput identification of human papillomavirus genotypes. J Clin Microbiol 40:779-787

13. Muñoz N, Bosch FX, de Sanjosé S et al (2003) Epidemiologic classification of human papillomavirus types associated with cervical cancer. N Engl J Med 348:518-527

14. Doll R, Payne P, Waterhouse J (1966) Cancer incidence in five continents: a technical report. Springer-Verlag (for UICC), Berlin

15. Franceschi S, Rajkumar R, Snijders PJF et al (2005) Papillomavirus infection in rural women in southern India. $\mathrm{Br} \mathrm{J}$ Cancer 92:601-606
16. Dai M, Bao YP, Li N et al (2006) Human papillomavirus infection in Shanxi Province, People's Republic of China: a population-based study. Br J Cancer 95:96-101

17. Li LK, Dai M, Clifford GM et al (2006) Human papillomavirus infection in Shenyang City, People's Republic of China: a population-based study. Br J Cancer 95:1593-1597

18. Wu RF, Dai M, Qiao YL et al (2007) Human papillomavirus infection in women in Shenzhen City, People's Republic of China, a population typical of recent Chinese urbanisation. Int $\mathrm{J}$ Cancer 121:1306-1311

19. Dondog B, Clifford GM, Vaccarella S et al (2008) Human papillomavirus infection in Ulaanbaatar, Mongolia: a population-based study. Cancer Epidemiol Biomarkers Prev 17:17311738

20. Keita N, Clifford GM, Koulibaly M et al (2009) HPV infection in women with and without cervical cancer in Conakry, Guinea. Br J Cancer 101:865-870

21. Anh PT, Hieu NT, Herrero R et al (2003) Human papillomavirus infection among women in South and North Vietnam. Int J Cancer 104:213-220

22. Sukvirach S, Smith JS, Tunsakul S et al (2003) Population-based human papillomavirus prevalence in Lampang and Songkla, Thailand. J Infect Dis 187:1246-1256

23. De Vuyst H, Clifford GM, Li N, Franceschi S (2009) HPV infection in Europe. Eur J Cancer 45:2632-2639

24. Thomas JO, Herrero R, Omigbodun AA et al (2004) Prevalence of papillomavirus infection in women in Ibadan, Nigeria: a population-based study. Br J Cancer 90:638-645

25. Franceschi S, Herrero R, Clifford GM et al (2006) Variations in the age-specific curves of human papillomavirus prevalence in women worldwide. Int J Cancer 119:2677-2684

26. Castle PE, Schiffman M, Herrero R et al (2005) A prospective study of age trends in cervical human papillomavirus acquisition and persistence in Guanacaste, Costa Rica. J Infect Dis 191:1808-1816

27. Khan MJ, Castle PE, Lorincz AT et al (2005) The elevated 10year risk of cervical precancer and cancer in women with human papillomavirus (HPV) type 16 or 18 and the possible utility of type-specific HPV testing in clinical practice. J Natl Cancer Inst 97:1072-1079

28. Vaccarella S, Franceschi S, Herrero R et al (2006) Sexual behavior, condom use and HPV: pooled analysis of the International Agency for Research on Cancer HPV Prevalence Surveys. Cancer Epidemiol Biomarkers Prev 15:326-333

29. Bardin A, Vaccarella S, Clifford GM et al (2008) Human papillomavirus infection in women with and without cervical cancer in Warsaw, Poland. Eur J Cancer 44:557-564

30. Smith JS, Lindsay L, Hoots B et al (2007) Human papillomavirus type distribution in invasive cervical cancer and high-grade cervical lesions: a meta-analysis update. Int J Cancer 121:621-632

31. Agurto I, Arrossi S, White S et al (2005) Involving the community in cervical cancer prevention programs. Int $\mathrm{J}$ Gynaecol Obstet 89(Suppl 2):S38-S45

32. Murillo R, Almonte M, Pereira A et al (2008) Cervical cancer screening programs in Latin America and the Caribbean. Vaccine 26(Suppl 11):L37-L48

33. Garland SM, Cuzick J, Domingo EJ et al (2008) Recommendations for cervical cancer prevention in Asia Pacific. Vaccine 26(Suppl 12):M89-M98 